\title{
Organizing principles of cortical layer 6
}

\section{Farran Briggs*}

Laboratory of Dr. W. M. Usrey, Center for Neuroscience, Division of Biological Sciences, University of California, Davis, CA, USA

\section{Edited by:}

Rafael Yuste, Columbia University, USA

\section{Reviewed by:}

Gilad Silberberg, Nobel Institute for

Neurophysiology, Karolinska Institute,

Sweden

Alex M. Thomson, University of

London, UK

\section{${ }^{*}$ Correspondence:}

Farran Briggs, Center for

Neuroscience, University of California,

Davis, 1544 Newton Court, Davis, CA

95618, USA.

e-mail: fbriggs@ucdavis.edu
Neurons in the deepest layer of mammalian cerebral cortex are morphologically and physiological diverse and are situated in a strategic position to modulate neuronal activity locally and in other structures. The variety of neuronal circuits within which layer 6 neurons participate differs across species and cortical regions. However even amidst this diversity, common organizational features emerge. Examination of the anatomical and physiological characteristics of different classes of layer 6 neuron, each specialized to participate in distinct circuits, provides insight into the functional contributions of layer 6 neurons toward cortical information processing.

Keywords: layer 6, cortex, morphology, physiology, circuit

\section{INTRODUCTION}

One of the most obvious distinguishing features of mammalian cerebral cortex is its laminar structure. While the size and sub-divisions of the cortical lamina vary across different areas and species, mammalian cortex contains six main layers. Neurons within these layers often differ in both their cellular morphology, i.e. the shape and distribution of their axonal and dendritic arborizations, and their physiological properties. The deepest cortical layer, layer 6 , is located in a strategic position within the local cortical architecture because it receives afferent input and provides output to other brain regions. Interestingly, layer 6 also contains the richest diversity of morphological cell types. However, neurophysiological studies have paid little attention to layer 6 compared to its more superficial counterparts, possibly because it is more difficult to access (e.g. given the depth limitations of in vivo imaging techniques). Another reason layer 6 remains elusive is it has proven difficult to define functionally, a probable outcome of its heterogeneity.

While the population of neuronal cell types within layer 6 is quite heterogeneous, neurons can be grouped into broad categories based on the laminar distributions of their local dendrites and axons. One class of excitatory pyramidal neurons project local dendrites and axons to layer 4 , the primary termination zone for thalamocortical afferents to sensory cortex. Through these arborizations, this class of layer 6 neuron is positioned to receive inputs from and provide outputs to neurons in layer 4, establishing the foundation for a reciprocal connectivity loop between these two layers. A second class of excitatory pyramidal neurons project local dendrites and axons to layer 5 , a major source of cortical output, thus allowing for reciprocal connectivity between neurons in layers 5 and 6 . A third, more heterogeneous group includes excitatory layer 6 neurons with local projections largely restricted to layer 6 . This group includes non-pyramidal neurons with spiny stellate-like morphology, non-vertically oriented pyramidal neurons, bipolar/fusiform neurons, and other odd-shaped cells. While these unusual neurons represent a minority of layer 6 neurons, their general presence is conserved across mammalian species and cortical areas. A final group of layer 6 neurons includes a variety of morphologically and physiologically distinct inhibitory cells. Inhibitory neurons within layer 6 are interneurons with local arborizations generally restricted to layer 6 , suggesting that these neurons mainly inhibit their neighbors.

Anatomical and physiological evidence suggests that neurons of these various classes participate in distinct cortical circuits mediating the flow of information into, within, and out of the home cortical area. By comparing examinations of layer 6 neurons in a variety of species and cortical areas using a variety of different technical approaches, this review is intended to provide some insight into the common organizational features of cortical layer 6 . Included within the sections below are descriptions of the known anatomy, physiology, and circuitry of each distinct cell class within layer 6 with some emphasis placed on excitatory pyramidal neurons in primary sensory cortical areas since more is known about these populations. Throughout, similarities and differences across cortical areas and species will be highlighted, while maintaining an underlying current of common organizational principles.

\section{ANATOMY}

The most striking characteristic unique to cortical layer 6 is its large diversity of neuronal cell types. In contrast to the other cortical lamina, which contain pyramidal neurons and, in some cases, spiny stellate neurons that vary subtly in somal size and arborization pattern, layer 6 contains multiple distinct classes of pyramidal neurons defined by their apical dendritic arborization patterns, a broad category of oddly shaped excitatory neurons, and a variety of inhibitory neurons (see for example Lund et al., 1979, 1988; Tombol, 1984; Ferrer et al., 1986a,b; Prieto and Winer, 1999; Thomson and Bannister, 2003; Callaway, 2004; Douglas and Martin, 2004; Andjelic et al., 2009).

The most characterized neurons in layer 6 are short and tall pyramidal neurons, which make up the majority of excitatory cells in layer 6 (Ferrer et al., 1986a,b; Lund, 1988; Prieto and Winer, 1999). Short pyramidal neurons target layer 4 with their axonal 
and dendritic arbors and rarely send apical dendrites higher than the bottom of layer 3 (Gilbert and Wiesel, 1979; Lund et al., 1979; Ferrer et al., 1986a,b; Katz, 1987; Lund, 1988; Usrey and Fitzpatrick, 1996; Wiser and Callaway, 1996; Zhang and Deschenes, 1997; Hirsch et al., 1998; Prieto and Winer, 1999; Bannister et al., 2002; Brumberg et al., 2003; Douglas and Martin, 2004; Zarrinpar and Callaway, 2006). This projection pattern is established early in development (Callaway and Lieber, 1996). Tall and/or horizontally projecting pyramidal neurons often have apical dendrites extending up to layer 1, but usually restrict the majority of apical dendritic branches to layer 5. Often tall pyramidal neurons also display long laterally projecting axons that terminate in layers 6 or 5, giving rise to the name 'horizontal' or 'lateral' cells (Lund et al., 1979; Ferrer et al., 1986a,b; Katz, 1987; Lund, 1988; Usrey and Fitzpatrick, 1996; Wiser and Callaway, 1996; Zhang and Deschenes, 1997; Hirsch et al., 1998; Prieto and Winer, 1999; Bannister et al., 2002; Douglas and Martin, 2004; Zarrinpar and Callaway, 2006). Within these two pyramidal cell classes there are often a number of distinct variations (Lund, 1988; Wiser and Callaway, 1996; Zhang and Deschenes, 1997; Prieto and Winer, 1999; Brumberg et al., 2003; Zarrinpar and Callaway, 2006). In primate primary visual cortex, for example, there are at least 8 different types of pyramidal neuron $(5$ cell types display short pyramidal morphology and 3 cell types display tall pyramidal morphology), each defined by local dendritic and axonal arborization patterns targeting specific sub-lamina (Wiser and Callaway, 1996; Briggs and Callaway, 2001). For the purposes of this review, all layer 6 cell types that preferentially target layer 4 will be referred to as 'short' while those neuronal types with tall apical dendrites and/or horizontal projections targeting layer 5 will be referred to as 'tall' layer 6 pyramidal neurons.

Based on anatomical observations of short and tall pyramidal neurons in primary visual cortex (V1) of cat and primate, these cells are hypothesized to participate in reciprocal circuits with layer 4 and layer 5, respectively (Figure 1; see for review, Callaway, 2004; Douglas and Martin, 2004). Within the larger framework of a proposed canonical microcircuit for a cortical column, these reciprocal circuits are thought to play a lesser role in local information processing compared to the robust connections from layer 4 to layer $2 / 3$ and between layers 2/3 and 5 (Douglas and Martin, 2004). Some connections to and from layer 6 do indeed appear to be weaker and/or sparser than other connections along the cortical hierarchy (Blasdel et al., 1985; Staiger et al., 2000; Briggs and Callaway, 2001; Thomson and Bannister, 2003; Binzegger et al., 2004; Callaway, 2004; Stepanyants et al., 2008; Llano and Sherman, 2009), however it is likely that this population may play an important role in regulating the flow of cortical information. Short and tall layer 6 neurons

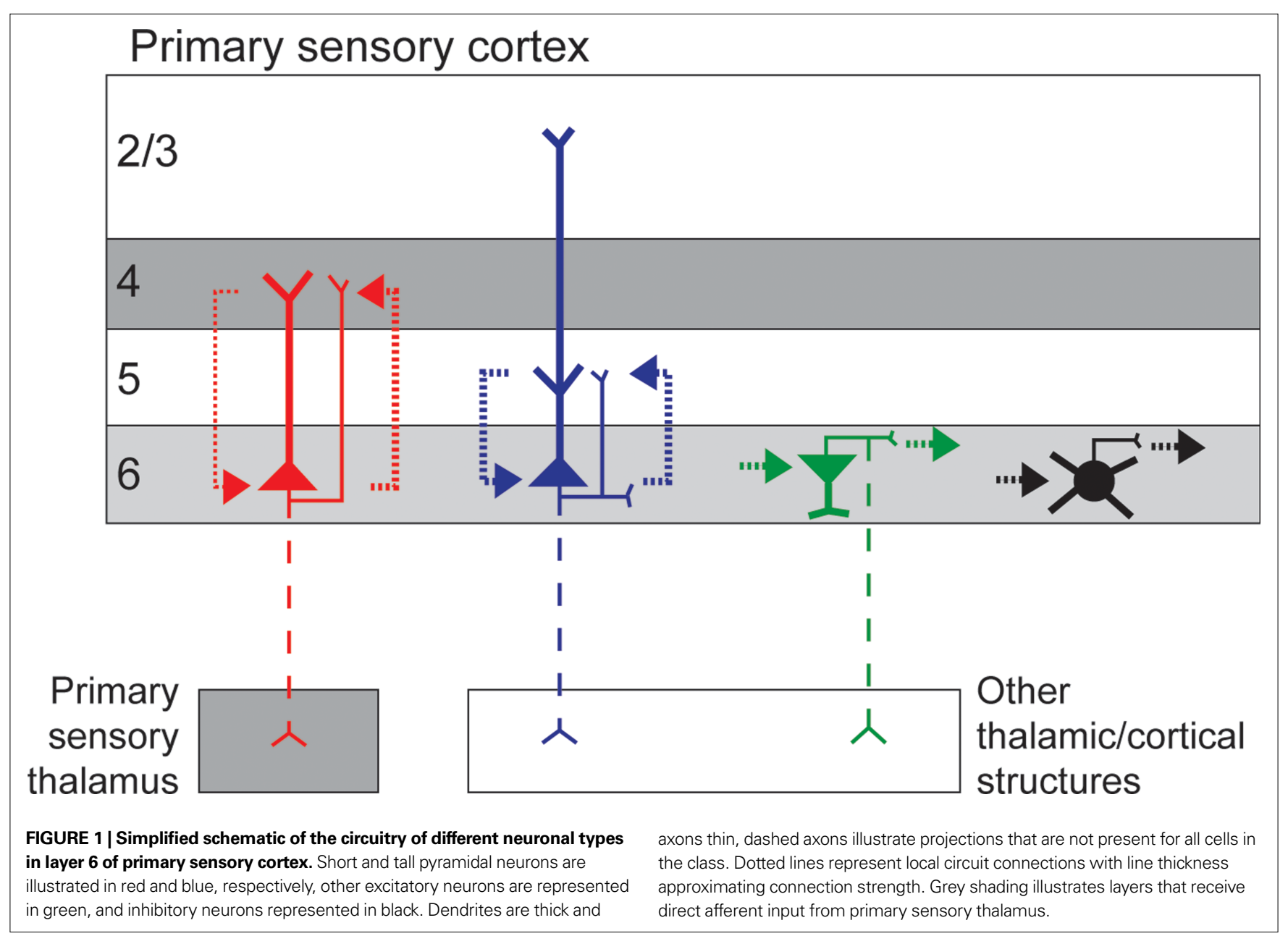


are located in a strategic position to sample inputs from and provide outputs to most local cortical layers, various sub-cortical structures, and other cortical areas. Therefore, while the influence of these layer 6 neurons may be subtle, it is far-reaching.

In addition to the varieties of tall and short pyramidal neurons, layer 6 contains a diverse group of other glutamatergic neurons, most of which restrict their local arborizations to layer 6 (Figure 1). There are many pyramidal neurons in layer 6 with apical dendritic orientations other than vertical (toward the pial surface) including upside-down, horizontal, and bent-over neurons with sideways somata and apical dendrites that turn up to $90^{\circ}$ to become vertical when approaching the pial surface (Tombol, 1984; Prieto and Winer, 1999; Joshi, 2007; Briggs et al., 2009). Non-vertically oriented pyramidal neurons are located in the deepest portion of layer 6 and often project dendrites into the white matter (Tombol, 1984; Prieto and Winer, 1999; Andjelic et al., 2009; Briggs et al., 2009). Physiological evidence suggests that these unusual pyramidal neurons are regular spiking, glutamatergic neurons (Andjelic et al., 2009), some of which make reciprocal connections to contralateral cortical areas (Karayannis et al., 2007).

Other non-pyramidal excitatory neurons in layer 6 include spiny stellate neurons located in the upper half of layer 6 , some of which project axons to the thalamus (Tombol, 1984; Zhang and Deschenes, 1997; Prieto and Winer, 1999; Briggs et al., 2009). Spiny stellate neurons in layer 6 may display adaptive spiking properties (Andjelic et al., 2009). Differently shaped bipolar or fusiform neurons (vertical, horizontal, multi-polar) reside in different portions of layer 6: vertical fusiform neurons are located in the upper tier of layer 6 and can project dendrites as high as layer 4 while horizontal fusiform neurons are encountered only in the deepest part of layer 6 (Tombol, 1984; Prieto and Winer, 1999; Andjelic et al., 2009). Sub-populations of these glutamatergic fusiform neurons project to other cortical regions, ipsilateral and contralateral, and/ or sub-cortical structures (Tombol, 1984; Zhang and Deschenes, 1997; Prieto and Winer, 1999; Karayannis et al., 2007; Andjelic et al., 2009). Aside from vertical fusiform neurons in the upper portion of layer 6 , most fusiform neurons extend dendritic and axonal arborizations within layer 6 and/or into the white matter (Tombol, 1984; Prieto and Winer, 1999; Andjelic et al., 2009).

Layer 6 contains another unique type of excitatory pyramidallike neuron, termed Meynert cells. These neurons have unusually large somata, prominent tall apical dendrites, and are located at the border between layers 5 and 6 in V1 (Chan-Palay et al., 1974; Fries and Distel, 1983; Lund, 1988). The local axons of Meynert cells extend up to many millimeters laterally and display small terminal clusters optimized for targeting specific cortical domains (Rockland and Knutson, 2001; Li et al., 2003). Axonal projections outside the home cortical area target the superior colliculus and other cortical areas (Fries et al., 1985; Lund, 1988). The unusual asymmetric shape of Meynert cells in V1 have inspired a number of interesting hypotheses for their role in visual processing, however the function of this distinct class of layer 6 neuron remains elusive.

Less is known quantitatively about the population of inhibitory neurons in layer 6 , however evidence suggests that layer 6 contains a large diversity of inhibitory neurons (Tombol, 1984; Ferrer et al., 1986a,b; Lund et al., 1988; Prieto et al., 1994; Thomson and Bannister, 2003; Kumar and Ohana, 2008). Studies of the proportion of inhibitory neurons in layer 6 of different cortical areas in different species have estimated values between 10 and 20\% (Prieto et al., 1994; Zhang and Deschenes, 1997). A number of distinct inhibitory cell types have been identified in layer 6. Basket and basket-like inhibitory neurons, sometimes called multi-polar neurons, are encountered most in the literature (see Lund et al., 1988; Prieto et al., 1994; Prieto and Winer, 1999; Thomson and Bannister, 2003). Basket neurons are located in the upper portion of layer 6 with dendrites descending throughout layer 6 and axons that can extend laterally below the layer 5/6 border and/or up into layer 4 (Lund et al., 1988; Prieto et al., 1994; Kumar and Ohana, 2008). Axon terminals of basket neurons often form clusters and target somata and proximal arborizations of pyramidal neurons and possibly Meynert cells (Lund, 1988; Lund et al., 1988; Thomson and Bannister, 2003). There are likely many different basket cell types, defined by morphological specializations, spiking dynamics, and neuro-chemical markers (see Thomson and Bannister, 2003; Kumar and Ohana, 2008). Other inhibitory neurons in layer 6 include chandelier cells, with similar arborization patterns and axonal targets to basket neurons (Lund et al., 1988; Thomson and Bannister, 2003); double-bouquet neurons with arborizations extending into layer 5 (Lund et al., 1988); and Martinotti cells, identified by axonal projections up to layer 1 (Prieto et al., 1994; Thomson and Bannister, 2003). Double-bouquet and Martinotti cells contact dendrites of spiny (excitatory) and/or smooth (inhibitory) target neurons (Prieto et al., 1994; Thomson and Bannister, 2003). Based on their morphological and physiological heterogeneity, different types of inhibitory neuron in layer 6 are likely to participate in specialized local circuits. While some inhibitory layer 6 neurons (e.g. basket and Martinotti cells) contact neurons outside of layer 6, most inhibitory neurons in layer 6 likely supply local inhibition to particular types of layer 6 excitatory neuron (Figure 1).

\section{OUTSIDE CONNECTIONS}

Certain populations of layer 6 neurons receive input from structures outside the home cortical area in addition to their local cortical inputs. A sub-population of short pyramidal neurons sample thalamic afferent input arriving from primary sensory thalamic nuclei via their dendritic branches in layers 4 and 6, the termination zones for sensory thalamic afferents (Hendrickson et al., 1978; Martin and Whitteridge, 1984; Lund, 1988; Bannister et al., 2002; Briggs and Usrey, 2007; Da Costa and Martin, 2009). Evidence from cat and monkey V1 suggests that the proportion of layer 6 cells that receive suprathreshold input from the LGN may differ across species with cat V1 containing a higher proportion of thalamocortical-recipient layer 6 neurons compared to primate (Ferster and Lindstrom, 1983; Briggs and Usrey, 2007). The exact proportion and morphological identification of thalamocorticalrecipient neurons in layer 6 of different species are not known. However a recent study in cat area 17 revealed low numbers of thalamocortical synaptic contacts mainly targeting the basal dendrites of corticothalamic neurons (Da Costa and Martin, 2009). Layer 6 is also a termination zone for input from the claustrum and from other cortical areas (LeVay and Sherk, 1981; Felleman and Van Essen, 1991; Casagrande and Kaas, 1994), however, again, 
the specific cell types and proportions of recipient neurons are not known. In rodent frontal cortex, odd-shaped excitatory neurons in layer 6 receive callosal input from contralateral cortices and, in some cases, send reciprocal projections to contralateral cortices (Karayannis et al., 2007).

In primary sensory cortical areas, neurons projecting back to primary sensory thalamic nuclei reside entirely within layer 6 (see for review Sherman and Guillery, 2005). Corticothalamic neurons targeting sensory thalamic nuclei send axon collaterals to the reticular nucleus, setting up a disynaptic inhibitory projection onto target thalamic neurons (Sherman and Guillery, 2005; Lam and Sherman, 2010). Other layer 6 neurons target different sub-cortical structures including higher-order thalamic nuclei such as the pulvinar nucleus, the superior colliculus, the claustrum, and/or other cortical regions (see for review, Ferrer et al., 1986a,b; Lund, 1988; Felleman and Van Essen, 1991; Casagrande and Kaas, 1994; Sherman and Guillery, 2005; Sincich and Horton, 2005). Interestingly, neuronal populations targeting different regions outside the home cortical area appear to be non-overlapping (see for example, LeVay and Sherk, 1981; Katz, 1987; Conley and Raczkowski, 1990; Usrey and Fitzpatrick, 1996). As a consequence, axonal projections outside of the home cortical area differ between short and tall pyramidal neurons. For example, in the most studied primary sensory cortical areas such as V1 (area 17) of cats and monkeys, and somatosensory or barrel cortex (S1) of rodents, corticothalamic neurons targeting primary sensory thalamic structures are short pyramids, while neurons projecting to other sub-cortical structures and/or cortical areas are tall pyramids (Gilbert and Kelly, 1975; LeVay and Sherk, 1981; Katz, 1987; Usrey and Fitzpatrick, 1996; Zhang and Deschenes, 1997; Kumar and Ohana, 2008; for similar findings in mouse $\mathrm{V} 1$ and cat and mouse primary auditory cortex, see Brumberg et al., 2003, and Prieto and Winer, 1999; Llano and Sherman, 2009). Even within the sub-classes of pyramidal neurons targeting outside structures, there appears to be heterogeneity. For example, corticothalamic neurons targeting specific lamina or regions of primary thalamic nuclei reside in different sub-laminar zones within layer 6 (Conley and Raczkowski, 1990; Fitzpatrick et al., 1994; Usrey and Fitzpatrick, 1996; Zhang and Deschenes, 1997; Deschenes et al., 1998). Recent evidence also suggests that corticothalamic neurons in V1 consist of functionally distinct cell types based on physiological measurements of axon conduction velocity and receptive field properties (Briggs and Usrey, $2005,2007,2009)$. In the monkey visual system, three distinct physiological types of corticogeniculate neuron have been characterized, each with specific relationships to the feedforward magnocellular, parvocellular, and koniocellular processing streams (Briggs and Usrey, 2009). Ongoing work suggests that the corticogeniculate pathway consists of multiple anatomically distinct neuronal types, including short pyramidal neurons, as previously described, and a minority of odd-shaped glutamatergic cells (see Briggs et al., 2009).

\section{SPECIES SPECIFICITY}

While maintaining a large diversity of neuronal cell types in layer 6 appears to be an evolutionary precedent, there is evidence in favor of some species-specific variations. There are a greater number of distinctly defined morphological cell types in primate layer 6 compared to other species (compare Wiser and Callaway, 1996; Briggs and Callaway, 2001 to Katz, 1987; Zhang and Deschenes, 1997; Zarrinpar and Callaway, 2006; and see Lund et al., 1979; Ferrer et al., 1986b; Callaway, 2004). For example, horizontally projecting neurons are more prominent in primate sensory cortices compared to other species (see Ferrer et al., 1986b), possibly a consequence of the sophisticated organization of primate cortex into functionally discrete columnar modules (Malach et al., 1993). Corticothalamic neurons in primate sensory cortices constitute a smaller proportion of layer 6 neurons, however they are more morphologically heterogeneous compared to those of cat and rodent (compare Conley and Raczkowski, 1990; Fitzpatrick et al., 1994; Usrey and Fitzpatrick, 1996; Wiser and Callaway, 1996 to Gilbert and Kelly, 1975; Katz, 1987; Zhang and Deschenes, 1997; Prieto and Winer, 1999; Kumar and Ohana, 2008). It is also likely that primates differ from other species in terms of the proportion of layer 6 neurons receiving primary sensory thalamic afferent input and/or the efficacy of such input (compare Bullier and Henry, 1980; Briggs and Usrey, 2007 to Ferster and Lindstrom, 1983; Martin and Whitteridge, 1984; Da Costa and Martin, 2009). Thus while cat area 17, rodent S1, and primate $\mathrm{V} 1$ are all highly specialized cortical areas, primary sensory cortex of primates may contain additional specificity indicative of more advanced cognitive processing.

\section{CIRCUITRY/PHYSIOLOGY}

Morphological analyses of axonal and dendritic projections can inform predictions about the different types of inputs to and outputs from any given neuronal cell type. Such anatomical predictions provide a model framework, such as the canonical circuit, which generates hypotheses that can be tested using physiological methods. A number of experimental techniques have been utilized to probe cortical circuitry, each with inherent advantages and disadvantages. As these techniques are refined and combined with exciting advances in genetic, viral, and imaging technologies, further elucidation of the physiology and circuitry of identified neuronal cell types will soon be forthcoming (see for example Nikolenko et al., 2008; Callaway, 2009; Petreanu et al., 2009).

\section{RECEPTIVE FIELD PHYSIOLOGY}

Elucidating the physiological responses of cortical neurons to sensory stimuli necessitates recording from intact preparations. Such in vivo methodologies are less amenable to morphological identification of recorded neurons. However, many studies have succeeded in determining the laminar location of recorded single units providing a picture of the physiological properties of layer 6 neurons. Correlated with the morphological diversity observed in layer 6 is a diversity of physiological properties. In V1 of cats, rabbits and primates, layer 6 contains both simple and complex cells with broadly distributed visual response characteristics (Harvey, 1978; Gilbert and Wiesel, 1979; Tsumoto and Suda, 1980; Swadlow and Weyand, 1987; Hawken et al., 1988, 1996; Anderson et al., 1993; Grieve and Sillito, 1995; Hirsch et al., 1998; Johnson et al., 2001; Ringach et al., 2002; Gur et al., 2005; Briggs and Usrey, 2005, 2007, 2009). Distinct physiological response profiles have also been described for layer 6 neurons in rodent S1 (Kwegyir-Afful and Simons, 2009). Studies that have directly correlated receptive field physiology with recorded neuronal morphology provide support for the notion that distinct morphological cell types in layer 6 express distinct physiological phenotypes (see for example, Gilbert and Wiesel, 1979; Martin and Whitteridge, 1984; Anderson et al., 1993; Hirsch et al., 1998; Bannister et al., 2002). 


\section{CIRCUITRY 1: SHORT PYRAMIDS CONNECT WITH LAYER 4 AND THE THALAMUS}

A more accessible system for studying local cortical circuitry within smaller (<500 microns), columnar domains or modules is the living tissue slice preparation. Obvious limitations of the slice preparation are the removal of inputs to and outputs from the isolated section, including longer-range lateral connections from neurons in the same cortical region (see Stepanyants et al., 2009). In living slices, whole-cell patch clamp recordings can be made from multiple individual neurons simultaneously (see for example, Brown and Hestrin, 2009; Lefort et al., 2009), enabling precise measurements of the strength and adaptation characteristics of connecting synapses. Alternatively, optical activation of presynaptic neurons can map a wider range of inputs onto simultaneously recorded postsynaptic cells. By combining insights gained from multiple whole-cell recordings and photostimulation studies of morphologically identified layer 6 neurons, it becomes clear that layer 6 neurons participate in a number of distinct circuits.

Functional inputs onto short pyramidal neurons in layer 6 arise primarily from layers 4 and 6 (Staiger et al., 2000; Briggs and Callaway, 2001; Zarrinpar and Callaway, 2006; Lefort et al., 2009), consistent with anatomical observations of axon/dendrite overlap within layers 4 and 6 (Lund, 1988; Yabuta and Callaway, 1998). The local axons of short pyramidal neurons primarily target layer 4, contacting both excitatory (stellate and/or pyramidal) and inhibitory cells (Blasdel et al., 1985; Lund, 1988; Binzegger et al., 2007; Lee and Sherman, 2009). While most of these projections are columnar, some extend outside the home cortical column in an indiscriminate manner with respect to ocular dominance columns (Blasdel et al., 1985; Wiser and Callaway, 1997; Binzegger et al., 2007). Short pyramidal axons utilize type I (excitatory) terminals to target dendritic regions of layer 4 neurons that are more distal compared to the target regions of thalamocortical afferents (Ahmed et al., 1994; Richardson et al., 2009). Furthermore, layer 6 cell synapses onto layer 4 neurons are quite numerous (Ahmed et al., 1994), utilize both ionotropic and metabotropic glutamate receptors (Lee and Sherman, 2009), and generate small EPSCs that are facilitating to paired-pulse stimulation (Tarczy-Hornoch et al., 1999). These axonal and synaptic properties are consistent with a modulatory role for layer 6 neurons in regulating layer 4 activity (Sherman and Guillery, 1998; Lee and Sherman, 2009).

In addition to participating in reciprocal circuits with layer 4 neurons, some short pyramidal neurons in layer 6 also participate in similar reciprocal circuits with primary sensory thalamic nuclei (see Briggs and Usrey, 2007, 2008). Corticothalamic axons projecting to primary sensory thalamic nuclei also utilize type I axon terminals to target distal dendrites of thalamic relay cells in multiple thalamic layers (Robson, 1983; Claps and Casagrande, 1990). Because corticothalamic neurons do not appear to dramatically alter the physiological response properties (e.g. receptive field physiology) of individual thalamic neurons, the corticothalamic pathway is thought to be more modulatory in nature (see Briggs and Usrey, 2008). It is important to consider, however, that while the influence of layer 6 on neurons in layer 4 and the thalamus may be subtle, it is notable that the same population of layer 6 neurons have direct access to both the input source (thalamus) and the input termination zone (layer 4 ). With such access, short layer 6 neurons may control the flow of feedforward information to the cortex.

\section{CIRCUITRY 2: TALL PYRAMIDS CONNECT WITH LAYER 5}

Across many species and modalities, the most prominent local functional input to layer 6 originates in layer 5, consistent with the canonical framework (Blasdel et al., 1985; Staiger et al., 2000; Briggs and Callaway, 2001; Binzegger et al., 2004; Douglas and Martin, 2004; Zarrinpar and Callaway, 2006; Weiler et al., 2008; Llano and Sherman, 2009). Layer 5 neurons receive robust local inputs from all cortical layers, including layer 6 , possibly conveyed by axons of tall pyramidal neurons (Thomson and Bannister, 2003; Binzegger et al., 2004; Callaway, 2004; Briggs and Callaway, 2005; Weiler et al., 2008; Llano and Sherman, 2009). Evidence suggests that tall pyramids differ from short pyramidal neurons in spike width and adaptation, and synaptic EPSP amplitude, variance, and paired-pulse response (Bannister et al., 2002; Mercer et al., 2005; Bremaud et al., 2007; Kumar and Ohana, 2008). These results, in combination with anatomical evidence, suggest that the connection between layers 5 and 6 may be mediated predominantly by tall pyramidal neurons. Because layer 5 neurons can transmit output signals to subcortical and other cortical areas (Felleman and Van Essen, 1991; Casagrande and Kaas, 1994; Sherman and Guillery, 2005; Sincich and Horton, 2005), tall pyramidal neurons in layer 6 may exert some influence on these output signals leaving layer 5 .

\section{CIRCUITRY 3: SUPERFICIAL LAYER INPUT, CONNECTIONS WITHIN LAYER 6, AND INHIBITORY CIRCUITS}

There may be some significant species-specific differences in the local circuitry of layer 6 neurons involving connections with the superficial layers. In primate V1, distinct cell types receive different patterns of input from the sub-divisions of the superficial layers (Briggs and Callaway, 2001). Interestingly, superficial layer input is seldom, if ever, observed in rodent cortices (Staiger et al., 2000; Zarrinpar and Callaway, 2006; Weiler et al., 2008; Lefort et al., 2009; Llano and Sherman, 2009). Additionally, some populations of layer 6 neurons in primate $\mathrm{V} 1$ project local axons to the superficial layers and physiological studies have identified weak inputs from layer 6 to superficial neurons (Usrey and Fitzpatrick, 1996; Sawatari and Callaway, 2000; Yabuta et al., 2001). In contrast, layer 6 provides little to no input to superficial neurons in rodent cortices (Dantzker and Callaway, 2000; Barbour and Callaway, 2008; Weiler et al., 2008; Lefort et al., 2009; Petreanu et al., 2009). How circuits connecting layer 6 with the superficial layers contribute to local processing in primates compared to other species remains a mystery.

In addition to inputs from layer 4 or layer 5 , most layer 6 neurons also receive inputs from other layer 6 neurons (Staiger et al., 2000; Briggs and Callaway, 2001; Binzegger et al., 2004; Zarrinpar and Callaway, 2006; Llano and Sherman, 2009). Given the large heterogeneity in the population of excitatory and inhibitory neurons with arborizations restricted to layer 6 , it is tempting to speculate that these populations mediate a number of within layer 6 circuits. Little is know about the local circuitry involving the more rare and unusual neuronal types within layer 6 so their role in cortical processing remains unclear. The potential for large connectivity among neighboring layer 6 neurons begs the question of 
whether there is crosstalk between the short and tall pyramidal cell populations. Limiting communication between these cell classes could be an optimal strategy for maintaining separation between the modulatory influence of layer 6 on thalamocortical communication (via short pyramidal neurons) and the driving influence on cortical outputs (via tall pyramidal neurons). To what extent these divergent circuits share information, or inhibit one another, remains an unresolved question.

The circuits involving inhibitory neurons in layer 6 have yet to be described in detail. Based on the anatomical observation that most inhibitory cell types within layer 6 restrict their arbors to layer 6 (Lund et al., 1988; Prieto et al., 1994), it is tempting to hypothesize that most layer 6 inhibitory neurons serve to inhibit their neighbors within the same layer. However, there is evidence to suggest that inhibitory neurons in layer 6 participate in local circuits involving other cortical lamina. Some basket cells in layer 6 display similar axonal projection patterns as short pyramidal neurons, targeting layer 4 preferentially (Lund et al., 1988; Prieto et al., 1994; Kumar and Ohana, 2008). These neurons could, therefore, participate in reciprocal connectivity with layer 4 , analogous to short pyramidal neurons. In rodents, layer 6 pyramidal neurons do not receive excitatory input from layer $2 / 3$ (see above); however both pyramidal and inhibitory layer 6 neurons do receive inhibitory input from the superficial layers (see Gonchar and Burkhalter, 1999; Zarrinpar and Callaway, 2006). Additionally, the action potential and synaptic kinetics of inhibitory neurons in layer 6 may vary in a cell type-specific manner and also differ from those of other cortical inhibitory neurons (Bremaud et al., 2007; Kumar and Ohana, 2008), suggestive of their participation in a variety of local circuits.

\section{SUMMARY}

Cortical layer 6 is composed of a morphologically heterogeneous population of neurons. Each unique cell type is likely to express a distinct physiological phenotype, a function of specialized local,

\section{REFERENCES}

Ahmed, B., Anderson, J. C., Douglas, R. J., Martin, K. A. C., and Nelson, J. C. (1994). Polyneuronal innervation of spiny stellate neurons in cat visual cortex. J. Comp. Neurol. 341, 39-49.

Anderson, J. C., Martin, K. A. C., and Whitteridge, D. (1993). Form, function, and intracortical projections of neurons in the striate cortex of the monkey Macacus nemestrinus. Cereb. Cortex 3, 412-420.

Andjelic, S., Gallopin, T., Cauli, B., Hill, E. L., Roux, L., Badr, S., Hu, E., Tamas, G., and Lambolez, B. (2009). Glutamatergic nonpyramidal neurons from neocortical layer VI and their comparison with pyramidal and spiny stellate neurons. J. Neurophysiol. 101, 641-654.

Bannister, N. J., Nelson, J. C., and Jack, J. J. B. (2002). Excitatory inputs to spiny cells in layers 4 and 6 of cat striate cortex. Philos. Trans. R. Soc. London 357, 1793-1808.
Barbour, D. L., and Callaway, E. M. (2008). Excitatory local connections of superficial neurons in rat auditory cortex. J. Neurosci. 28, 11174-11185.

Binzegger, T., Douglas, R. J., and Martin, K. A. C. (2004). A quantitative map of the circuit of cat primary visual cortex. J. Neurosci. 24, 8441-8453.

Binzegger, T., Douglas, R. J., and Martin, K. A. C. (2007). Stereotypical bouton clustering of individual neurons in cat primary visual cortex. J. Neurosci. 27, 12242-12254.

Blasdel, G. G., Lund, J. S., and Fitzpatrick, D. (1985). Intrinsic connections of macaque striate cortex: axonal projections of cells outside lamina 4C. J. Neurosci. 5, 3350-3369.

Bremaud, A., West, D. C., and Thomson, A. M. (2007). Binomial parameters differ across neocortical layers and with different classes of connections in adult rat and cat neocortex. Proc. Natl. Acad. Sci. U.S.A. 104, 14134-14139.

subcortical, and/or intracortical inputs. A common organizational principle of layer 6 across mammalian species is the presence of two classes of pyramidal neuron, short and tall pyramids, which participate in specialized cortical circuits. Short pyramidal neurons are reciprocally connected to neurons in layer 4 and/or in primary sensory thalamic nuclei and the features of their connections suggest they modulate the activity of their target neurons (see LeVay, 1986; Sherman and Guillery, 1998; Callaway, 2004; Briggs and Usrey, 2008). While their influence may be subtle, short pyramidal neurons are undoubtedly a crucial component of sensory processing because they are positioned to influence the feedforward flow of sensory input to the cortex. Tall pyramidal neurons may also be involved in reciprocal circuitry within and outside their home cortical area through connections with cortical layer 5 . In this manner, tall pyramids may serve to control the output flow of information from one cortical region to the next.

In addition to short and tall pyramidal neurons, layer 6 contains a large diversity of odd-shaped excitatory neurons and a variety of inhibitory cells. While most neurons within these populations are likely to be involved in local connectivity within layer 6, anatomical and physiological evidence suggests that each of these unique cell types likely play distinct roles in cortical information processing.

Taken together, the studies reviewed here all point to a pivotal role for layer 6 in controlling the flow of information into and out of cortical networks. Much work remains in order to understand the functional properties of each and every distinct cell type within layer 6; however new technologies hold great promise toward elucidating this complex cortical layer.

\section{ACKNOWLEDGMENTS}

I would like to thank Drs. S. M. Sherman, W. M. Usrey, and P. Adams for insightful comments on this manuscript. This work was supported by NIH grants EY018683 and EY013588.

Briggs, F., and Callaway, E. M. (2001) Layer-specific input to distinct cell types in layer 6 of monkey primary visual cortex. J. Neurosci. 21, 3600-3608.

Briggs, F., and Callaway, E. M. (2005). Laminar patterns of local excitatory input to layer 5 neurons in macaque primary visual cortex. Cereb. Cortex 15, 479-488.

Briggs, F., Roby, K., Callaway, E. M., and Usrey, W. M. (2009). Morphological classification of corticogeniculate neurons in the macaque monkey. Abstr. Soc. Neurosci. 848.20.

Briggs, F., and Usrey, W. M. (2005) Temporal properties of feedforward and feedback pathways between thalamus and visual cortex in the ferret. Thal. Rel. Sys. 3, 133-139.

Briggs, F., and Usrey, W. M. (2007). A fast, reciprocal pathway between the lateral geniculate nucleus and visual cortex in the macaque monkey. J. Neurosci. 27, 5431-5436.
Briggs, F., and Usrey,W.M.(2008).Emerging views of corticothalamic function. Curr. Opin. Neurobiol. 18, 403-407.

Briggs, F., and Usrey, W.M. (2009). Parallel processing in the corticogeniculate pathway of the macaque monkey. Neuron 62, 135-146.

Brown, S. P., and Hestrin, S. (2009). Intracortical circuits of pyramidal neurons reflect their longrange axonal targets. Nature 457, 1133-1136.

Brumberg, J. C., Hamzei-Sichani, F., and Yuste, R. (2003). Morphological and physiological characterization of layer VI corticofugal neurons of mouse primary visual cortex. J. Neurophysiol. 89, 2854-2867.

Bullier, J., and Henry, G. H. (1980). Ordinal position and afferent input of neurons in monkey striate cortex. J. Comp. Neurol. 193, 913-935.

Callaway, E. M. (2004). Cell types and local circuits in primary visual cortex of the macaque monkey. In The Visual 
Neurosciences, Vol. 1, L. Chalupa and J. Werner, eds (Cambridge, MA, MIT Press), pp. 680-694.

Callaway, E. M. (2009). Transneuronal circuit tracing with neurotropic viruses. Curr. Opin. Neurobiol. doi: 10.1016/j.conb.2009.03.007.

Callaway, E. M., and Lieber, J. L. (1996). Development of axonal arbors of layer 6 pyramidal neurons in ferret primary visual cortex. J. Comp. Neurol. 376, 295-305.

Casagrande, V. A., and Kaas, J. H. (1994). The afferent, intrinsic, and efferent connections of primary visual cortex in primates. In Cerebral Cortex, Vol. 10, A. Peters and K. S. Rockland, eds (Plenum Press, New York, NY), pp. 201-249.

Chan-Palay, V., Palay, S. L., and BillingsGagliardi, S. M. (1974). Meynert cells in the primate visual cortex. $J$. Neurocytol. 3, 631-658.

Claps, A., and Casagrande, V. A. (1990). The distribution and morphology of corticogeniculate axons in ferrets. Brain Res. 530, 126-129.

Conley, M., and Raczkowski, D. (1990). Sublaminar organization within layer VI of the striate cortex in Galago. J. Comp. Neurol. 302, 425-436.

Da Costa, N. M., and Martin, K. A. C. (2009). Selective targeting of the dendrites of corticothalamic cells by thalamic afferents in area 17 of the cat. J. Neurosci. 29, 13919-13928.

Dantzker, J. L., and Callaway, E. M. (2000). Laminar sources of synaptic input to cortical inhibitory interneurons and pyramidal neurons. Nat. Neurosci. 3, 701-707.

Deschenes, M., Veinante, P., and Zhang, Z.-W. (1998). The organization of corticothalamic projections: reciprocity versus parity. Brain Res. Rev. 28, 286-308

Douglas, R. J., and Martin, K.A. C. (2004). Neuronal circuits of the neocortex. Annu. Rev. Neurosci. 27, 419-451.

Felleman, D. J., and Van Essen, D. C. (1991). Distributed hierarchical processing in the primate cerebral cortex. Cereb. Cortex 1, 1-47.

Ferrer, I., Fabregues, I., and Condom, E. (1986a). A golgi study of the sixth layer of the cerebral cortex. I. The lissencephalic brain of Rodentia, Lagomorpha, Insectivora and Chiroptera. J. Anat. 145, 217-234.

Ferrer, I., Fabregues, I., and Condom, E. (1986b). A golgi study of the sixth layer of the cerebral cortex II. The gyrencephalic brain of Carnivora, Artiodactyla and Primates. J. Anat. 146, 87-104.

Ferster, D., and Lindstrom, S. (1983). An intracellular analysis of geniculocortical connectivity in area 17 of the cat. J. Physiol. 342, 181-215.
Fitzpatrick, D., Usrey, W. M., Schofield, B. R., and Einstein, G. (1994). The sublaminar organization of corticogeniculate neurons in layer 6 of macaque striate cortex. Vis. Neurosci. 11, 307-315.

Fries, W., and Distel, H. (1983). Large layer VI neurons of monkey striate cortex (Meynert cells) project to the superior colliculus. Proc. R. Soc. Lond., B, Biol. Sci. 219, 53-59.

Fries, W., Keizer, K., and Kuypers, H. G. (1985). Large layer VI cells in macaque strate cortex (Meynert cells) project to both superior colliculus and prestriate visual area V5. Exp. Brain Res. 58, 613-616.

Gilbert, C. D., and Kelly, J. P. (1975). The projections of cells in different layers of the cat's visual cortex. J. Comp. Neurol. 163, 81-106.

Gilbert, C. D., and Wiesel, T. N. (1979). Morphology and intracortical projections of functionally characterized neurons in the cat visual cortex. Nature 280, 120-125.

Gonchar, Y., and Burkhalter, A. (1999). Connectivity of GABAergic calretininimmunoreactive neurons in rat primary visual cortex. Cereb. Cortex 9 , 683-696.

Grieve, K. L., and Sillito, A. M. (1995). Differential properties of cells in the feline primate visual cortex providing the corticofugal feedback to the lateral geniculate nucleus and visual claustrum. J. Neurosci. 15, 4868-4874.

Gur, M., Kagan, I., and Snodderly, D. M. (2005). Orientation and direction selectivity of neurons in V1 of alert monkeys: Functional relationships and laminar distributions. Cereb. Cortex 15, 1207-1221.

Harvey, A. R. (1978). Characteristics of corticothalamic neurons in area 17 of the cat. Neurosci. Lett. 7, 177-181.

Hawken, M. J., Parker, A. J., and Lund, J. S. (1988). Laminar organization and contrast sensitivity of directionselective cells in the striate cortex of the old world monkey. J. Neurosci. 8, 3541-3548.

Hawken, M. J., Shapley, R. M., and Grosof, D. H. (1996). Temporal-frequency selectivity in monkey visual cortex. Vis. Neurosci. 13, 477-492.

Hendrickson, A. E., Wilson, J. R., and Ogren, M. P. (1978). The neuroanatomical oganization of pathways between the dorsal lateral geniculate nucleus and visual cortex in the old world and new world primates. $J$. Comp. Neurol. 182, 123-136.

Hirsch, J. A., Gallagher, C. A., Alonso, J. -M., and Martinez, L. M. (1998). Ascending projections of simple and complex cells in layer 6 of the cat striate cortex. J. Neurosci. 18, 8086-8094.

Johnson, E. N., Hawken, M. J., and Shapley, R. M. (2001). The spatial transformation of color in the primary visual cortex of the macaque monkey. Nat. Neurosci. 4, 409-416.

Joshi, S. (2007). Structure-Function Relations in Macaque V1. Ph.D. Dissertation, New York University, New York.

Karayannis, T., Huerta-Ocampo, I., and Capogna, M. (2007). GABAergic and pyramidal neurons of deep cortical layers directly receive and differentially integrate callosal input. Cereb. Cortex 17, 1213-1226.

Katz, L. C. (1987). Local circuitry of identified projection neurons in cat visual cortex brain slices. J. Neurosci. 7, 1223-1249.

Kumar, P., and Ohana, O. (2008). Interand intralaminar subcircuits of excitatory and inhibitory neurons in layer $6 a$ of the rat barrel cortex. J. Neurophys. 100, 1909-1922.

Kwegyir-Afful, E. E., and Simons, D. J. (2009). Subthreshold receptive field properties distinguish different classes of corticothalamic neurons in the somatosensory system. J. Neurosci. 29, 964-972.

Lam, Y-W., and Sherman, S. M. (2010). Functional organization of the somatosensory cortical layer 6 feedback to the thalamus. Cereb. Cortex 20 , 13-24.

Lee, C. C., and Sherman, S. M. (2009). Modulator property of the intrinsic cortical projection from layer 6 to layer 4. Front. Syst. Neurosci. 3:3. doi:10.3389/neuro.06.003.2009.

Lefort, S., Tomm, C., Sarria, J. -C. F., and Petersen, C. C. H. (2009). The excitatory neuronal network of the C2 barrel column in mouse primary somatosensory cortex. Neuron 61 , 301-316.

LeVay, S. (1986). What layer 6 tells layer 4. Nature 320, 310-311.

LeVay, S., and Sherk, H. (1981). The visual claustrum of the cat I. Structure and connections. J. Neurosci. 1, 956-980.

Li, H., Fukuda, J., Tanifuji, M., and Rockland, K. S. (2003). Intrinsic collaterals of layer 6 Meynert cells and functional columns in primate V1. Neuroscience 120, 1061-1069.

Llano, D. A., and Sherman, S. M. (2009). Differences in intrinsic properties and local network connectivity of identified layer 5 and layer 6 adult mouse auditory corticothalamic neurons support a dual corticothalamic projection hypothesis. Cereb. Cortex 19, 2810-2826.

Lund, J. S. (1988). Anatomical organization of macaque monkey striate visual cortex. Annu. Rev. Neurosci. 11, 253-288.

Lund, J. S., Hawken, M. J., and Parker, A. J. (1988). Local circuit neurons of macaque monkey striate cortex: II.
Neurons of laminae 5B and 6. J. Comp. Neurol. 276, 1-29.

Lund, J. S., Henry, G. H., MacQueen, C. L., and Harvey, A. R. (1979). Anatomical organization of the primary visual cortex (area 17) of the cat. A comparison with area 17 of the macaque monkey. J. Comp. Neurol. 184, 599-618.

Malach, R., Amir, Y., Harel, M., and Grinvald, A. (1993). Relationship between intrincis connections and functional architecture revealed by optical imaging and in vivo targeted biocytin injections in primate striate cortex. Proc. Natl. Acad. Sci. U.S.A. 90 10469-10473.

Martin, K. A. C., and Whitteridge, D. (1984). Form, function and intracortical projections of spiny neurons in the striate visual cortex of the cat. $J$. Physiol. 353, 463-504.

Mercer, A., West, D. C., Morris, O. T., Kirchhecker, S., Kerkhoff, J. E., and Thomson, A. M. (2005). Excitatory connections made by presynaptic cortico-cortical pyramidal cells in layer 6 of the neocortex. Cereb. Cortex 15, 1485-1496.

Nikolenko, V., Watson, B. O., Araya, R., Woodruff,A., Peterka, D. S., and Yuste, R. (2008). SLM microscopy: scanless two-photon imaging and photostimulation with spatial light modulators. Front. Neural Circuits.2:5. doi: 10.3389/neuro.04.005.2008.

Petreanu, L., Mao, T., Sternson, S. M., and Svoboda, K. (2009). The subcellular organization of neocortical excitatory connections. Nature 457, 1142-1145.

Prieto, J. J., Peterson, B. A., and Winer, J. A. (1994). Morphology and spatial distribution of GABAergic neurons in cat primary auditory cortex (A1). J. Comp. Neurol. 344, 349-382.

Prieto, J. J., and Winer, J. A. (1999). Layer VI in cat primary auditory cortex: Golgi study and sublaminar origins of projection neurons. J. Comp. Neurol. 404, 332-358.

Richardson, J. R., Blaundon, J. A., Bayazitov, I. T., and Zakharenko, S. S. (2009).Connectivity patterns revealed by mapping of active inputs on dendrites of thalamorecipient neurons in the auditory cortex. J. Neurosci. 29, 6406-6417.

Ringach, D. L., Shapley, R. M., and Hawken, M. J. (2002). Orientation selectivity in macaque V1: Diversity and laminar dependence. J. Neurosci. 22, 5639-5651.

Robson, J. A. (1983). The morphology of corticofugal axons to the dorsal lateral geniculate nucleus in the cat. J. Comp. Neurol. 216, 89-103.

Rockland, K. S., and Knutson, T. (2001). Axon collaterals of Meynert cells diverge over large portions of area 
V1 in the macaque monkey. J. Comp. Neurol. 441, 134-147.

Sawatari, A., and Callaway, E. M. (2000). Diversity of cell type specificity of local excitatory connections to neurons in layer $3 \mathrm{~B}$ of monkey primary visual cortex. Neuron 25, 459-471.

Sherman, S. M., and Guillery, R. W. (1998). On the actions that one nerve cell can have on another: Distringuishing "drivers" from "modulators”. Proc. Natl. Acad. Sci. U.S.A. 95, 7121-7126.

Sherman, S.M., and Guillery, R.W. (2005). Exploring the Thalamus and its Role in Cortical Function. 2nd Edn. Boston, MA, MIT Press.

Sincich, L. C., and Horton, J. C. (2005). The circuitry of V1 and V2: Integration of color, form, and motion. Annu. Rev. Neurosci. 28, 303-326.

Staiger, J. F., Kotter, R., Zilles, K., and Luhmann, H. J. (2000). Laminar characteristics of functional connectivity in rat barrel cortex revealed by stimulation with caged-glutamate. Neurosci. Res. 37, 49-58.

Stepanyants, A., Hirsch, J. A., Martinez, L. M., Kisvarday, Z. F., Ferecsko, A. S., and Chklovskii, D. B. (2008). Local potential connectivity in cat primary visual cortex. Cereb. Cortex 18, 13-28.
Stepanyants, A., Martinez, L. M., Ferecsko, A. S., and Kisvarday, Z. F. (2009). The fraction of short- and long-range connections in the visual cortex. Proc. Natl. Acad. Sci. U.S.A. 106, 3555-3560.

Swadlow, H.A., and Weyand, T. G. (1987). Corticogeniculate neurons, corticotectal neurons, and suspected interneurons in visual cortex of awake rabbits: receptive-field properties, axonal properties, and effects of EEG arousal. J. Neurophysiol. 57, 977-1001.

Tarczy-Hornoch, K., Martin, K. A. C., Stratford, K. J., and Jack, J. J. B. (1999). Intracortical excitation of spiny neurons in layer 4 of cat striate cortex in vitro. Cereb. Cortex 9, 833-843.

Thomson, A. M., and Bannister, A. P. (2003). Interlaminar connections in the neocortex. Cereb. Cortex 13, 5-14.

Tombol, T. (1984). Layer VI cells. In Cerebral Cortex, Vol. 1, A. Peters and E. G. Jones, eds (Plenum Press, New York, NY), pp. 479-519.

Tsumoto, T., and Suda, K. (1980). Three groups of cortico-geniculate neurons and their distribution in binocular and monocular segments of cat striate cortex. J. Comp. Neurol. 193, 223-236.
Usrey, W. M., and Fitzpatrick, D. (1996). Specificity in the axonal connections of layer VI neurons in tree shrew striate cortex: evidence for distinct granular and supragranular systems. J. Neurosci. 16, 1203-1218.

Weiler, N., Wood, L., Yu, J., Solla, S. A., and Shepherd, G. M. G. (2008). Topdown laminar organization of the excitatory network in motor cortex. Nat. Neurosci. 11, 360-366.

Wiser, A. K., and Callaway, E. M. (1996). Contributions of individual layer 6 pyramidal neurons to local circuitry in macaque primary visual cortex. $J$. Neurosci. 16, 2724-2739.

Wiser, A. K., and Callaway, E. M. (1997). Ocular dominance columns and local projections of layer 6 pyramidal neurons in macaque primary visual cortex. Vis. Neurosci. 14, 241-251.

Yabuta, N. H., and Callaway, E. M (1998). Functional streams and local connections of layer $4 \mathrm{C}$ neurons in primary visual cortex of the macaque monkey. J. Neurosci. 18 9489-9499.

Yabuta, N. H., Sawatari, A., and Callaway, E. M. (2001). Two functional channels from primary visual cortex to dorsal visual cortical areas. Science 292, 297-300.
Zarrinpar, A., and Callaway, E. M. (2006). Local connections to specific types of layer 6 neurons in the rat visual cortex. J. Neurophysiol. 95, 1751-1761.

Zhang, Z.-W., and Deschenes, M. (1997). Intracortical axonal projections of lamina VI cells of the primary somatosensory cortex in the rat: a single-cell labeling study. J. Neurosci. 17, 6365-6379.

Conflict of Interest Statement: The author declares that the research was conducted in the absence of any commercial or financial relationships that could be construed as a potential conflict of interest.

Received: 02 June 2009; paper pending published: 26 December 2009; accepted: 27 January 2010; published online: 12 February 2010

Citation:Briggs F (2010) Organizingprinciples of cortical layer 6. Front. Neural Circuits 4:3. doi: 10.3389/neuro.04.003.2010 Copyright $(2010$ Briggs. This is an openaccess article subject to an exclusive license agreement between the authors and the Frontiers Research Foundation, which permits unrestricted use, distribution, and reproduction in any medium, provided the original authors and source are credited. 